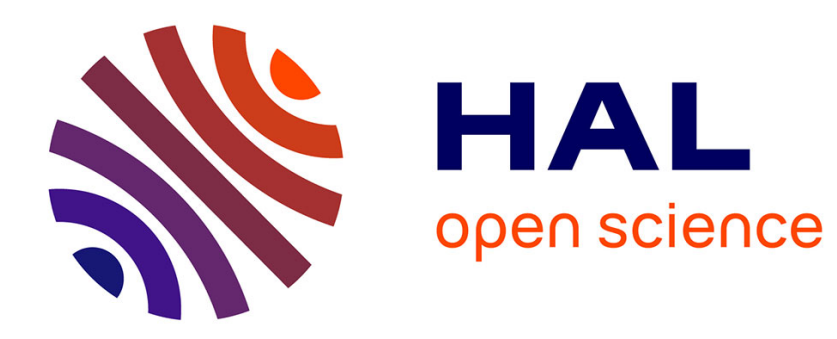

\title{
Obstacle and boundary determination from scattering data
}

\author{
Gen Nakamura, Mourad Sini
}

\section{To cite this version:}

Gen Nakamura, Mourad Sini. Obstacle and boundary determination from scattering data. 2007. hal-00136017

\author{
HAL Id: hal-00136017 \\ https://hal.science/hal-00136017 \\ Preprint submitted on 12 Mar 2007
}

HAL is a multi-disciplinary open access archive for the deposit and dissemination of scientific research documents, whether they are published or not. The documents may come from teaching and research institutions in France or abroad, or from public or private research centers.
L'archive ouverte pluridisciplinaire HAL, est destinée au dépôt et à la diffusion de documents scientifiques de niveau recherche, publiés ou non, émanant des établissements d'enseignement et de recherche français ou étrangers, des laboratoires publics ou privés. 


\title{
Obstacle and boundary determination from scattering data.
}

\author{
Gen Nakamura*and Mourad Sini ${ }^{\dagger}$
}

March 11, 2007

\begin{abstract}
In this paper, we are concerned with the identification of complex obstacles from the scattering data for the acoustic problem. The complex obstacle is characterized by its shape and the boundary values of the impedance coefficient. We establish point-wise formulas which can be used to reconstruct the shape of the obstacle and give explicitly the values of the surface impedance as a function of the far field. In addition, these formulas enable us to distinguish and recognize the coated and the non-coated parts of the obstacle.
\end{abstract}

\section{Introduction}

Let $D$ be a bounded domain of $\mathbb{R}^{m}, m \geq 3$, such that $\mathbb{R}^{m} \backslash \bar{D}$ is connected. In addition, we assume that its boundary $\partial D$ is of class $C^{2}$. Precisely, for every point $a \in \partial D$, there exist a rigid transformation of coordinates under which $a=0$ and a $C^{2}\left(B_{m-1}(0, r)\right)$-function $f$ such that

$$
f(0)=\frac{\partial f}{\partial x_{i}}(0)=0, i=1,2
$$

and

$$
D \cap B(0, r):=\left\{x \in B(0, r) ; x_{3}>f\left(x_{1}, x_{2}\right)\right\}
$$

in terms of the new coordinates where $B_{m-1}(0, r)$ and $B(0, r)$ are the $(m-1)$-dimensional and the $m$-dimensional balls of centers 0 with some radius $r>0$.

The propagation of time-harmonic acoustic fields in a homogeneous media is governed by the Helmholtz equation

$$
\Delta u+\kappa^{2} u=0 \quad \text { in } \quad \mathbb{R}^{m} \backslash \bar{D},
$$

where $\kappa$ is the real positive wave number. At the boundary of the scatterers we assume that the total field $u$ satisfies the impedance boundary condition

$$
\frac{\partial u}{\partial \nu}+i \lambda u=0 \text { on } \partial D_{I}
$$

with some function $\lambda$ on $\partial D$ and the Dirichlet condition

$$
u=0 \text { on } \partial D_{D}
$$

where $\partial D_{D}$ and $\partial D_{I}$ are open surfaces in $\partial D$ such that

$$
\partial D=\overline{\partial D_{I}} \cup \overline{\partial D_{D}} \text { and } \partial D_{I} \cap \partial D_{D}=\emptyset .
$$

\footnotetext{
*Department of Mathematics, Hokkaido University, Sapporo 060-0810, Japan.
}

${ }^{\dagger}$ Corresponding author. RICAM, Altenbergerstrasse 69, A 40-40, Linz, Austria. 
The unit normal $\nu$ on $\partial D$ is directed inside $D$. We assume that $\lambda$ is a Hölder continuous function of order $\beta \in(0,1]$ and $\lambda_{-}<\lambda(x)$ on $\partial D_{I}$ where $\lambda_{-}$is a positive constant. The part $\partial D_{I}$ is referred to by the coated part of $\partial D$ and $\partial D_{D}$ is the non-coated part as it is commonly used in the radar detection theory, see [5]. The obstacle $D$ is characterized by its shape, $\partial D_{D}, \partial D_{I}$ and the surface impedance distributed on $\partial D_{I}$. We call such obstacles complex obstacles.

Given an incident field $u^{i}$ which satisfies $\Delta u^{i}+\kappa^{2} u^{i}=0$ we look for a solution $u:=u^{i}+u^{s}$ of (1.2) and (1.3) where the scattered field $u^{s}$ is assumed to satisfy the Sommerfeld radiation condition

$$
\lim _{r \rightarrow \infty} r^{\frac{m-1}{2}}\left(\frac{\partial u^{s}}{\partial r}-i \kappa u^{s}\right)=0
$$

where $r=|x|$ and the limit is uniform with respect to all the directions $\hat{x}:=\frac{x}{|x|}$.

The mixed problem (1.2)-(1.3)-(1.4)-(1.5) is well posed. More generally, for $f \in H^{\frac{1}{2}}\left(\partial D_{D}\right)$ and $h \in H^{-\frac{1}{2}}\left(\partial D_{I}\right)$, there exists a unique solution $u \in H_{l o c}^{1}\left(R^{m} \backslash \bar{D}\right)$ of the mixed problem

$$
\left\{\begin{array}{l}
\left(\Delta+\kappa^{2}\right) u=0, \quad \text { in } R^{m} \backslash \bar{D} \\
u=f \quad \text { on } \partial D_{D}, \\
\frac{\partial u}{\partial \nu}+i \kappa \sigma u=h, \quad \text { on } \partial D_{I}, \\
\lim _{r \rightarrow \infty} r^{\frac{m-1}{2}}\left(\frac{\partial u}{\partial r}-i \kappa u\right)=0
\end{array}\right.
$$

and the solution satisfies

$$
\|u\|_{H^{1}\left(\Omega_{R} \cap\left(R^{m} \backslash \bar{D}\right)\right)} \leq C_{R}\left(\|f\|_{H^{1 / 2}\left(\partial D_{D}\right)}+\|h\|_{H^{-\frac{1}{2}}\left(\partial D_{I}\right)}\right)
$$

where $\Omega_{R}$ is a disk of radius $R$ and $C_{R}$ is positive constant depending on $R$, see [5] for more details.

It is well known (see [6]) that this reflected field satisfies the following asymptotic property,

$$
u^{s}(x)=\frac{e^{i \kappa r}}{r^{\frac{m-1}{2}}} u^{\infty}(\hat{x})+O\left(r^{-\frac{m+1}{2}}\right), \quad r \rightarrow \infty,
$$

where the function $u^{\infty}(\cdot)$ defined on the unit sphere $\mathbb{S}^{m}$ of $R^{m}$ is called the far-field associated to the incident field $u^{i}$. Taking particular incident fields given by the plane waves, $u^{i}(x, d):=e^{i \kappa d \cdot x}$, $d \in \mathbb{S}^{m}$, we define the far-field pattern $u^{\infty}(\hat{x}, d)$ for $(\hat{x}, d) \in \mathbb{S}^{m} \times \mathbb{S}^{m}$. Analogously, for an incident point source $\Phi(\cdot, z)$, where

$$
\Phi(x, y):=\frac{1}{(m-2) \sigma_{m}} \frac{e^{i \kappa|x-y|}}{|x-y|^{m-2}}, x \neq y, x, y \in \mathbb{R}^{m} .
$$

is the fundamental solution of $\Delta+\kappa^{2}$ in $\mathbb{R}^{m}$ and $\sigma_{m}$ is the surface of the unit sphere in $\mathbb{R}^{m}$, we denote the scattered field by $\Phi^{s}(\cdot, z)$ and its far field pattern by $\Phi^{\infty}(\cdot, z)$. The problem we are concerned with is the following

Complex obstacle reconstruction problem. Given $u^{\infty}(\cdot, \cdot)$ on $\mathbb{S}^{m} \times \mathbb{S}^{m}$ for the scattering problem (1.2) - (1.5) reconstruct the shape of the obstacle $D$, distinguish the coated part $\partial D_{D}$ from $\partial D_{I}$ and reconstruct the surface impedance $\lambda(x)$.

The uniqueness character of this problem is already known, see [5]. The part of the problem consisting of the effective detection of the shape of the obstacle $\partial D$ can be justified for instance via the linear sampling method, the factorization method or the probing methods ( the probe method or equivalently the singular sources method), see [17] for a review of these methods. Our goal in 
this paper is to show that not only the shape but the full complex obstacle can be reconstructed. Many efforts have been made regarding the determination of the surface impedance function $\lambda(x)$. We refer to the paper [11] where an optimization method has been proposed by assuming that the shape of the obstacle is known in advance. A different method is given in [7] where the authors first reduce the far field data to the near field data, and then from these near field data they propose a moment method to reconstruct $\lambda$. Another work is [3], see also [5], where the authors computed the $L^{\infty}$-norm of $\lambda$. As a consequence, if $\lambda$ is known to be constant, $\lambda=\lambda_{0}$, then they compute $\lambda_{0}$. All these works use a part or the total far field. We mention the work [2], where the authors use only one incident wave to detect $\lambda(x)$. Assuming that the whole surface $\partial D$ is coated, they first compute the total field and then use the impedance boundary condition to give the values of $\lambda(x)$. By the unique continuation, there is no open subset of $\partial D$ in which the normal derivative of the total field may vanish. However, there can be infinitely many points in $\partial D$ at which the total field vanishes. By avoiding these points, it is possible to reconstruct the value of $\lambda(x)$, and then by $\lambda \in C(\partial D)$, it is possible to know $\lambda$ on the whole $\partial D$. Hence this method cannot sample each point $x$ of $\partial D$ to obtain the value $\lambda(x)$. To remedy to this difficulty, the authors propose a regularization method.

We want to contribute to this problem by giving pointwise formulas to reconstruct fully the complex obstacle. Indeed, these formulas simultaneously reconstruct the shape of the obstacle, distinguish between the coated and the non coated parts and detect $\lambda(x)$ directly from the far field pattern defined on any small open part of the unit sphere $\mathbb{S}^{m}$.

To justify our formulas, we need to analyze the asymptotic behavior of the Green's function, of the mixed boundary problem, near $\partial D$. The impedance function $\lambda(x)$ appears in the asymptotic behavior of the imaginary part of this Green's function with respect to the source parameter $z$, see the proof of Proposition 3.1. In the 2 dimensional case the imaginary part of the corresponding Green's function is bounded with respect to the source parameter $z$. This is why we consider the $m$ dimensional case with $m>2$. For the 2 dimensional case, we need to use more singular sources to capture the values of the surface impedance. This has been analyzed in [13] and the corresponding formulas have been justified theoretically and tested numerically. We refer to that paper for more details on how the formulas are used numerically.

Regarding the stability issue for detecting the surface impedance, in case we know the shape, we cite the following results [12] and [18] where the authors use one incident wave and give interesting and optimal results. Another interesting question is to consider the stability of the complex obstacle. We leave this for future investigations.

The rest of the paper is organized as follows. In section 2, we present the results as Theorem 2.1. In section 3 , we give the proof of this theorem by splitting it into two propositions which we prove in section 3.1 and section 3.2 .

\section{Presentation of the results.}

It is well known (see [6]) that the scattered field associated with the Herglotz incident field $v_{g}^{i}:=v_{g}$ defined by

$$
v_{g}(x):=\int_{\mathbb{S}^{m}} e^{i \kappa x \cdot d} g(d) d s(d), \quad x \in \mathbb{R}^{m}
$$

whit $g \in L^{2}\left(\mathbb{S}^{m}\right)$ is given by

$$
v_{g}^{s}(x):=\int_{\mathbb{S}^{m}} u^{s}(x, d) g(d) d s(d), \quad x \in \mathbb{R}^{m} \backslash D,
$$


and its far field is given by

$$
v_{g}^{\infty}(\hat{x}):=\int_{\mathbb{S}^{m}} u^{\infty}(\hat{x}, d) g(d) d s(d), \quad \hat{x} \in \mathbb{S}^{m}
$$

We will need the following identity

$$
u^{\infty}(\hat{x}, d)=-\frac{1}{(m-2) \sigma_{m}} \int_{\partial D}\left\{\frac{\partial u^{s}(y, d)}{\partial \nu} e^{-i \kappa \hat{x} \cdot y}-\frac{\partial e^{-i \kappa \hat{x} \cdot y}}{\partial \nu} u^{s}(y, d)\right\} d s(y)
$$

given by using the Green's formula in $\mathbb{R}^{m} \backslash \bar{D}$ for $u^{s}(\cdot, d)$ and $\Phi(\cdot, y)$ and their asymptotic behavior at infinity (see [6], Theorem 2.5). The representation of the scattered field $\Phi^{s}(x, z)$ for $x, z \in \mathbb{R}^{m} \backslash \bar{D}$ is given by Green's formula

$$
\Phi^{s}(x, z)=-\int_{\partial D}\left\{\frac{\partial \Phi^{s}(y, z)}{\partial \nu(y)} \Phi(x, y)-\Phi^{s}(y, z) \frac{\partial \Phi(x, y)}{\partial \nu(y)}\right\} d s(y), \quad x, z \in \mathbb{R}^{3} \backslash \bar{D} .
$$

Let $a \in \partial D$ and a sequence of points

$$
\left(z_{p}\right)_{p \in \mathbb{N}} \subset \mathbb{R}^{m} \backslash \bar{D}
$$

such that $z_{p}$ tends to $a$. We consider the sequence of point sources $\Phi\left(\cdot, z_{p}\right)$. We set $D_{a}^{p}$ a $C^{2}-$ regular open set such that $\bar{D} \subset D_{a}^{p}, z_{p} \in \mathbb{R}^{n} \backslash \overline{D_{a}^{p}}$ for every $p \in \mathbb{N}$ and that the Dirichlet interior problem on $D_{a}^{p}$ is uniquely solvable. In this case, the Herglotz wave operator $H$ defined from $L^{2}\left(\mathbb{S}^{m}\right)$ to $L^{2}\left(\partial D_{a}^{p}\right)$ by

$$
H(g)(x):=v_{g}(x)=\int_{\mathbb{S} m} e^{i \kappa x \cdot d} g(d) d s(d)
$$

is injective, compact with dense range, see [6]. Hence by the Tikhonov regularization method, see [8] or [10], we can construct a sequence $g_{n}^{p}$ in $L^{2}\left(\mathbb{S}^{m}\right)$ such that for every $p$ fixed

$$
\left\|v_{g_{n}^{p}}-\Phi\left(\cdot, z_{p}\right)\right\|_{L^{2}\left(\partial D_{a}^{p}\right)} \rightarrow 0, \quad n \rightarrow \infty .
$$

Since both of $v_{g_{n}^{p}}$ and $\Phi\left(\cdot, z_{p}\right)$ satisfy the same Helmholtz equation in $D_{a}^{p},(2.16)$ implies that

$$
\left\|v_{g_{n}^{p}}-\Phi\left(\cdot, z_{p}\right)\right\|_{H^{\frac{1}{2}}(\partial D)} \rightarrow 0, \quad n \rightarrow \infty .
$$

Multiplying (2.12) by $\left.g_{n}^{p}(\hat{x}) g_{n}^{p}(d)\right)$ and integrating over $\mathbb{S}^{m}$, we have

$$
\begin{aligned}
& \int_{\mathbb{S}^{m}} \int_{\mathbb{S}^{m}} u^{\infty}(-\hat{x}, d) g_{n}^{p}(\hat{x}) g_{n}^{p}(d) d s(\hat{x}) d s(d) \\
= & -\frac{1}{(m-2) \sigma_{m}} \int_{\partial D}\left\{\int_{\mathbb{S}^{m}} \frac{\partial u^{s}(y, d)}{\partial \nu} g_{n}^{p}(d) d s(d) \cdot \int_{\mathbb{S}^{m}} e^{i \kappa \hat{x} \cdot y} g_{n}^{p}(\hat{x}) d s(\hat{x})\right. \\
& \left.-\int_{\mathbb{S}^{m}} \frac{\partial e^{i \kappa \hat{x} \cdot y}}{\partial \nu} g_{n}^{p}(\hat{x}) d s(\hat{x}) \cdot \int_{\mathbb{S}^{m}} u^{s}(y, d) g_{n}^{p}(d) d s(d)\right\} d s(y) \\
= & -\frac{1}{(m-2) \sigma_{m}} \int_{\partial D}\left\{\frac{\partial v_{g_{n}^{p}}^{s}}{\partial \nu}(y) v_{g_{n}^{p}}^{i}(y)+\frac{\partial v_{g_{n}^{p}}^{i}}{\partial \nu}(y) v_{g_{n}^{p}}^{s}(y)\right\} d s(y) .
\end{aligned}
$$


From (2.17), we have

$$
\begin{aligned}
& \lim _{n \rightarrow \infty} \int_{\mathbb{S}^{m}} \int_{\mathbb{S}^{m}} u^{\infty}(-\hat{x}, d) g_{n}^{p}(\hat{x}) g_{n}^{p}(d) d s(\hat{x}) d s(d) \\
= & -\frac{1}{(m-2) \sigma_{m}} \int_{\partial D} \frac{\partial\left(\Phi^{s}\right.}{\partial \nu(y)}\left(y, z_{p}\right) \Phi\left(y, z_{p}\right)-\Phi^{s}\left(y, z_{p}\right) \frac{\partial(\Phi}{\partial \nu(y)}\left(y, z_{p}\right) d s(y) \\
= & \frac{1}{(m-2) \sigma_{m}} \Phi^{s}\left(z_{p}, z_{p}\right)
\end{aligned}
$$

For the point $a \in \partial D$, we choose the sequence $\left(z_{p}\right)_{p \in \mathbb{N}}$ included in $C_{a, \theta}$, where $C_{a, \theta}$ is the cone with center $a$, angle $\theta \in\left[0, \frac{\pi}{2}\right)$ and axis $-\nu(a)$ where $\nu(a)$ is the unit normal of $\partial D$ directed inside $D$.

THEOREM 2.1 The surface of the obstacle can be localized via the formulas:

$$
\left|32 \pi^{2} \lim _{n \rightarrow \infty} \operatorname{Re} \int_{\mathbb{S}^{m}} \int_{\mathbb{S}^{m}} u^{\infty}(-\hat{x}, d) g_{n}^{p}(\hat{x}) g_{n}^{p}(d) d s(x) d s(d)\right|=\frac{1}{\left|\left(z_{p}-a\right) \cdot \nu(a)\right|}+O\left(\left|\ln \left(\left|z_{p}-a\right|\right)\right|\right),
$$

for $m=3$ and

$$
\begin{gathered}
\left|\frac{(m-2) \sigma_{m} 2^{2 m-2} \pi^{m-1}}{\sigma_{m-1}} \lim _{n \rightarrow \infty} \operatorname{Re} \int_{\mathbb{S}^{m}} \int_{\mathbb{S}^{m}} u^{\infty}(-\hat{x}, d) g_{n}^{p}(\hat{x}) g_{n}^{p}(d) d s(x) d s(d)\right|= \\
\frac{1}{\left|\left(z_{p}-a\right) \cdot \nu(a)\right|^{m-2}}+O\left(\left|z_{p}-a\right|^{3-m}\right),
\end{gathered}
$$

for $m>3$.

In addition, we have the following formulas for distinguishing the coated part from the noncoated part of the obstacle and for detecting the surface impedance:

I. The case $m=3$ :

I. 1. If $a \in \partial D_{I}$, then we have

$$
\lim _{p \rightarrow \infty}-8 \pi^{2} \frac{\lim _{n \rightarrow \infty} \operatorname{Im} \int_{\mathbb{S}^{m}} \int_{\mathbb{S}^{m}} u^{\infty}(-\hat{x}, d) g_{n}^{p}(\hat{x}) g_{n}^{p}(d) d s(\hat{x}) d s(d)}{\left.|\ln |\left(z_{p}-a\right) \cdot \nu(a)\right|^{s}}=\left\{\begin{array}{l}
\infty, \text { if } s \in[0,1), \\
\lambda(a) \text { if } s=1, \\
0 \text { if } s>1 .
\end{array}\right.
$$

I. 2. If $a \in \partial D_{D}$, then $\forall s>0$ we have:

$$
\lim _{p \rightarrow \infty} \frac{\lim _{n \rightarrow \infty} \operatorname{Im} \int_{\mathbb{S}^{m}} \int_{\mathbb{S}^{m}} u^{\infty}(-\hat{x}, d) g_{n}^{p}(\hat{x}) g_{n}^{p}(d) d s(\hat{x}) d s(d)}{\left.|\ln |\left(z_{p}-a\right) \cdot \nu(a)\right|^{s}}=0 .
$$

II. The case $m>3$ :

II. 1. If $a \in \partial D_{I}$, then we have

$$
\begin{gathered}
\lim _{p \rightarrow \infty} \frac{(m-2) \sigma_{m}(2 \pi)^{m-1}}{\sigma_{m-1}}\left(2\left(z_{p}-a\right) \cdot \nu(a)\right)^{m-3+s} \lim _{n \rightarrow \infty} \operatorname{Im} \int_{\mathbb{S}^{m}} \int_{\mathbb{S}^{m}} u^{\infty}(-\hat{x}, d) g_{n}^{p}(\hat{x}) g_{n}^{p}(d) d s(\hat{x}) d s(d)= \\
\left\{\begin{array}{l}
\infty, \text { if } s \in[0,1), \\
\lambda(a) \text { if } s=1, \\
0 \text { if } s>1 .
\end{array}\right.
\end{gathered}
$$


II. 2. If $a \in \partial D_{D}$, then $\forall s>0$ we have:

$$
\lim _{p \rightarrow \infty}\left|\left(z_{p}-a\right) \cdot \nu(a)\right|^{m-3+s} \lim _{n \rightarrow \infty} \operatorname{Im} \int_{\mathbb{S}^{m}} \int_{\mathbb{S}^{m}} u^{\infty}(-\hat{x}, d) g_{n}^{p}(\hat{x}) g_{n}^{p}(d) d s(\hat{x}) d s(d)=0 .
$$

REMARK 2.2 1. In the case $m=3$, from (2.22) and (2.23), we can localize the coated part of the obstacle by taking any $s \in(0,1)$, then taking $s=1$ in (2.22), we obtain the pointwise values of the surface impedance. Similarly we have the same conclusions for $m \geq 4$.

2. We stated the results by using the full far field pattern, i.e. $(\theta, d) \in \mathbb{S}^{m} \times \mathbb{S}^{m}$. We used this information to approximate the point sources $\Phi\left(\cdot, z_{p}\right)$ by Herglotz functions defined on the whole unit sphere $\mathbb{S}^{m}$. However this approximation is also justified if we define the Herglotz functions on any subsurface $\gamma \subset \mathbb{S}^{m}$ and hence the results of Theorem 2.1 are also valid if we replace $\mathbb{S}^{m}$ by any subsurface $\gamma \subset \mathbb{S}^{m}$.

\section{Proof of Theorem 2.1 .}

Let $\Gamma_{\lambda(a)}$ be a local Green's function satisfying:

$$
\left\{\begin{array}{l}
\Delta \Gamma_{\lambda(a)}=-\delta(x, z) \text { in } \mathbb{R}_{+}^{m} \\
\left(\frac{\partial \Gamma_{\lambda(a)}}{\partial \nu}+i \lambda(a) \Gamma_{\lambda(a)}\right)\left(x_{1}, x_{2}, \ldots, x_{m-1}, 0\right)=0
\end{array}\right.
$$

and $\Gamma_{D}$ defined by

$$
\Gamma_{D}(x, z):=\Gamma(x, z)-\Gamma\left(x^{*}, z\right)
$$

where $x=\left(x_{1}, x_{2}, \ldots, x_{m}\right), x^{*}=\left(x_{1}, x_{2}, \ldots,-x_{m}\right)$ and $\Gamma(x, z)=\frac{1}{(m-2) \sigma_{m}|x-z|^{m-2}}$.

It is clear that $\Gamma_{D}(x, z)$ satisfies

$$
\left\{\begin{array}{l}
\Delta \Gamma_{D}=-\delta(x, z) \text { in } \mathbb{R}_{+}^{m} \\
\Gamma_{D}\left(x_{1}, x_{2}, \ldots, x_{m-1}, 0\right)=0 .
\end{array}\right.
$$

We state the following propositions. Their proofs will be given in section 3.1 and section 3.2 respectively.

Proposition 3.1 The local Green function $\Gamma_{\lambda(a)}$ is given by

$$
\Gamma_{\lambda(a)}(x, z):=\Gamma(x, z)+\frac{1}{2(2 \pi)^{m-1}} \int_{\mathbb{R}^{m-1}} e^{i\left(x^{\prime}-z^{\prime}\right) \cdot \xi^{\prime}} e^{-\left(x_{m}+z_{m}\right)\left|\xi^{\prime}\right|} \frac{\left|\xi^{\prime}\right|+i \lambda(a)}{\left|\xi^{\prime}\right|\left(\left|\xi^{\prime}\right|-i \lambda(a)\right)} d \xi^{\prime},
$$

where $x^{\prime}=\left(x_{1}, x_{2}, \ldots, x_{m-1}\right)$ and $\xi^{\prime}=\left(\xi_{1}, \xi_{2}, \ldots, \xi_{m-1}\right)$.

In addition, we have the following asymptotics for the function $\left(\Gamma_{\lambda(a)}-\Gamma\right)(x, z)$ :

$$
\begin{gathered}
\operatorname{Re}\left(\Gamma_{\lambda(a)}-\Gamma\right)(z, z)=\frac{\sigma_{m-1}}{2(2 \pi)^{m-1}} \frac{1}{\left(2 z_{m}\right)^{m-2}}+O\left(\frac{1}{\left(z_{m}\right)^{m-3}}\right), \\
\lim _{z_{3} \rightarrow 0^{+}}-2 \pi \frac{\operatorname{Im}\left(\Gamma_{\lambda(a)}-\Gamma\right)(z, z)}{\ln \left(z_{3}\right)}=\lambda(a), \text { for } m=3
\end{gathered}
$$

and

$$
\lim _{z_{m} \rightarrow 0^{+}} \frac{(2 \pi)^{m-1}}{\sigma_{m-1}}\left(2 z_{m}\right)^{m-3} \operatorname{Im}\left(\Gamma_{\lambda(a)}-\Gamma\right)(z, z)=\lambda(a), \text { for } m>3 .
$$


Proposition 3.2 If $a \in \partial D_{I}$, then there exist $\delta(a)>0$ and $C>0$ such that

$$
\left|\Phi^{s}(x, z)-\left(\Gamma_{\lambda(a)}-\Gamma\right)(x, z)\right| \leq C\left\{\begin{array}{l}
|\ln | z-a||, \text { if } m=3, \\
\frac{1}{|z-a|^{m-3}} \text { if } m>3,
\end{array} \quad \text { for }(x, z) \in B_{+}(a, \delta(a)) \cap C_{a, \theta},\right.
$$

and

$\left|\operatorname{Im}\left(\Phi^{s}(x, z)-\left(\Gamma_{\lambda(a)}-\Gamma\right)(x, z)\right)\right| \mid \leq \frac{C}{|z-a|^{m-3-\beta}}+C$ for $m \geq 3$ and $(x, z) \in B_{+}(a, \delta(a)) \cap C_{a, \theta}$,

where $B_{+}(a, \delta(a)):=B(a, \delta(a)) \cap\left(\mathbb{R}^{3} \backslash D\right)$ and $B(a, \delta(a))$ is the ball of center $a$ and radius $\delta(a)$. Similarly, if $a \in \partial D_{D}$, we obtain (3.29) and (3.30) by replacing $\Gamma_{\lambda(a)}$ by $\Gamma_{D}$.

End of the proof of Theorem 2.1 .

1. Let $a \in \partial D_{I}$, i.e. we have the impedance boundary condition around $a$. By a rigid transformation of coordinates, we can assume that $a=(0,0,0, . ., 0)$. Using (2.19), Proposition 3.1 and Proposition 3.2 we obtain the formulas (2.20), (2.21), (2.22) and (2.24).

2. Let $a \in \partial D_{D}$, i.e. we have the Dirichlet boundary condition around $a$. Similarly, we can assume that $a=(0,0,0)$. Using (2.19), Proposition 3.2 and the fact that $\operatorname{Im} \Gamma_{D}=0=\operatorname{Im} \Gamma$ we obtain (2.23) and (2.25).

The rest of this section is devoted to prove Proposition 3.1 and Proposition 3.2.

\subsection{Proof of Proposition 3.1.}

We set $\Gamma_{\lambda(a)}(x, z):=\Gamma(x, z)+w(x, z)$, then $w(x, z)$ satisfies

$$
\left\{\begin{array}{l}
\Delta w(x, z)=0 \text { in } \mathbb{R}_{+}^{m} \\
\left(\partial_{x_{3}}+i \lambda(a)\right) w(x, z)=-\left(\partial_{x_{3}}+i \lambda(a)\right) \Gamma(x, z) \text { on } \partial \mathbb{R}_{+}^{m} .
\end{array}\right.
$$

The first part of this proposition is to show the following explicit form of $w(x, z)$.

LEMMA 3.3

$$
w(x, z)=\frac{1}{2(2 \pi)^{m-1}} \int_{\mathbb{R}^{m-1}} e^{i\left(x^{\prime}-z^{\prime}\right) \cdot \xi^{\prime}} e^{-\left(x_{m}+z_{m}\right)\left|\xi^{\prime}\right|} \frac{\left|\xi^{\prime}\right|+i \lambda(a)}{\left|\xi^{\prime}\right|\left(\left|\xi^{\prime}\right|-i \lambda(a)\right)} d \xi^{\prime} .
$$

Proof of Lemma 3.3.

We represent $w(x, z)$ using up going and down going operators $U_{ \pm}$

$$
w(x, z):=\left(U_{ \pm}\left(x_{m}\right) \phi\right)\left(x^{\prime}\right):=\frac{1}{(2 \pi)^{m-1}} \int_{\mathbb{R}^{m-1}} e^{i x^{\prime} \cdot \xi^{\prime} \mp x_{m}\left|\xi^{\prime}\right|} \hat{\phi}_{ \pm}\left(\xi^{\prime}, z\right) d \xi^{\prime}
$$

where $\hat{\phi}_{ \pm}$is the two dimensional Fourier transform of $\phi_{ \pm}$. The goal is to find $\phi_{ \pm}$, or $\hat{\phi}_{ \pm}$. We start by the corresponding representation of $\Gamma(x, z)$. We write

$$
\Gamma(x, z)=\left\{\begin{array}{l}
\Gamma_{+}(x, z) \text { in } x_{m}>z_{m}, \\
\Gamma_{-}(x, z) \text { in } x_{m}<z_{m}
\end{array}\right.
$$

then $\Delta \Gamma_{ \pm}=0$ in $\pm\left(x_{m}-z_{m}\right)>0$ with the transmission conditions

$$
\left\{\begin{array}{l}
\left.\Gamma_{+}\right|_{x_{m}=z_{m}+0}=\Gamma_{-}||_{x_{m}=z_{m}-0}, \\
\partial_{x_{m}} \Gamma_{+}\left|x_{x_{m}=z_{m}+0}-\partial_{x_{m}} \Gamma_{-}\right|_{x_{m}=z_{m}-0}=-\delta\left(x^{\prime}, z^{\prime}\right) .
\end{array}\right.
$$


Now we look for $\Gamma_{ \pm}$in the form

$$
\Gamma_{ \pm}(x, z)=U_{ \pm}\left(x_{m}-z_{m}\right) \psi_{ \pm}\left(x^{\prime}, z^{\prime}\right)
$$

and try to determine the potentials $\psi_{ \pm}$.

Clearly, from the definition of $U_{ \pm}$, we have

$$
\Delta \Gamma_{ \pm}=0 \text { in } \pm\left(x_{m}-z_{m}\right)>0
$$

and from the first equation of (3.34), we get

$$
\psi_{+}=\psi_{-} .
$$

Let us now consider the second equation of (3.34). We set

$$
(B f)\left(x^{\prime}\right):=\int_{\mathbb{R}^{m-1}} e^{i x^{\prime} \cdot \xi^{\prime}}(-|\xi|) \hat{f}\left(\xi^{\prime}\right) \bar{d} \xi^{\prime},
$$

then we deduce that

$$
\partial_{x_{m}} U_{ \pm}\left(x_{m}\right)= \pm B U_{ \pm}\left(x_{m}\right) .
$$

The point two of (3.34) implies that

$$
B \psi_{+}+B \psi_{-}=-\delta\left(x^{\prime}-z^{\prime}\right) .
$$

Taking the Fourier transform, we have

$$
-\left|\xi^{\prime}\right| \hat{\psi}_{+}-\left|\xi^{\prime}\right| \hat{\psi}_{-}=-e^{i z^{\prime} \cdot \xi^{\prime}}
$$

and combining (3.35) with (3.37), we end up with:

$$
\hat{\psi}_{ \pm}\left(\xi^{\prime}, z^{\prime}\right)=\frac{1}{2}\left|\xi^{\prime}\right|^{-1} e^{-i z^{\prime} \cdot \xi^{\prime}}
$$

Now, we go back to $w(x, z)$. We set $\phi_{ \pm}:=\phi$ in (3.32), i.e.

$$
w(x, z)=\left(U_{+}\left(x_{m}\right) \phi\right)\left(x^{\prime}, z\right),
$$

then from (3.36) we have

$$
\left.\left(\partial_{x_{3}}+i \lambda(a)\right) w\right|_{x_{m}=0}=B \phi+i \lambda(a) \phi,
$$

because $U_{+}(0) \phi=\phi$. By Fourier transform, the right hand side of (3.39) becomes

$$
-\left|\xi^{\prime}\right| \hat{\phi}\left(\xi^{\prime}\right)+i \lambda(a) \hat{\phi}\left(\xi^{\prime}\right) .
$$

By similar computations for the fundamental solution $\Gamma(x, z)$, we have

$$
\begin{gathered}
-\left.\left(\partial_{x_{m}} \Gamma_{-}+i \lambda(a) \Gamma_{-}\right)\right|_{x_{m}=0}=-\left.\left(-B \Gamma_{-}+i \lambda(a) \Gamma_{-}\right)\right|_{x_{m}=0} \\
=-\left(-U_{-}\left(-z_{m}\right) B \psi_{-}+i \lambda(a) U_{-}\left(-z_{m}\right) \psi_{-}\right)\left(x^{\prime}\right)
\end{gathered}
$$

because

$$
B U_{ \pm}=U_{ \pm} B
$$


The Fourier transform of (3.41) is

$$
-e^{-z_{m}\left|\xi^{\prime}\right|}\left(\left|\xi^{\prime}\right|+i \lambda(a)\right) \hat{\psi}_{-}\left(\xi^{\prime}, z^{\prime}\right)
$$

hence combining (3.40) with (3.42), we obtain

$$
\hat{\phi}\left(\xi^{\prime}, z\right)=\frac{\left|\xi^{\prime}\right|+i \lambda(a)}{\left|\xi^{\prime}\right|-i \lambda(a)} e^{-z_{m}\left|\xi^{\prime}\right|} \hat{\psi}_{-}\left(\xi^{\prime}, z^{\prime}\right) .
$$

Using (3.35), we have

$$
\hat{\phi}\left(\xi^{\prime}, z\right)=\frac{1}{2} \frac{\left|\xi^{\prime}\right|+i \lambda(a)}{\left|\xi^{\prime}\right|\left(\left|\xi^{\prime}\right|-i \lambda(a)\right)} e^{-z_{m}\left|\xi^{\prime}\right|} e^{-i z^{\prime} \cdot \xi^{\prime}}
$$

Finally (3.32) becomes

$$
w(x, z)=\frac{1}{2(2 \pi)^{m-1}} \int_{\mathbb{R}^{m-1}} e^{i\left(x^{\prime}-z^{\prime}\right) \cdot \xi^{\prime}} e^{-\left(x_{m}+z_{m}\right)\left|\xi^{\prime}\right|} \frac{\left|\xi^{\prime}\right|+i \lambda(a)}{\left|\xi^{\prime}\right|\left(\left|\xi^{\prime}\right|-i \lambda(a)\right)} d \xi^{\prime} .
$$

Next we deal with the second part of the proposition. From Lemma 3.3, we have

$$
w(z, z)=\frac{1}{(2 \pi)^{m-1}} \int_{\mathbb{R}^{m-1}} e^{-\left(2 z_{m}\right) \mid \xi^{\prime}} \frac{1}{2} \frac{\left|\xi^{\prime}\right|+i \lambda(a)}{\left|\xi^{\prime}\right|\left(\left|\xi^{\prime}\right|-i \lambda(a)\right)} d \xi^{\prime} .
$$

We start with the case $m=3$. Using polar coordinates, we write

$$
w(z, z)=\frac{1}{4 \pi^{2}} \int_{0}^{2 \pi} d \theta \int_{0}^{\infty} e^{-2 z_{3} r} \frac{1}{2 r}\left(1+\frac{2 i \lambda(a)}{r-i \lambda(a)}\right) r d r=\frac{1}{2 \pi} \int_{0}^{\infty} e^{-2 z_{3} r} \frac{1}{2 r}\left(1+\frac{2 i \lambda(a)}{r-i \lambda(a)}\right) r d r .
$$

After some computations, we obtain

$$
\operatorname{Re} w(z, z)=\frac{1}{8 \pi z_{3}}+O(1) .
$$

Similarly we obtain

$$
2 \pi \operatorname{Im} w(z, z)=\lambda(a)\left[-\ln (\lambda(a))+\int_{0}^{\infty} e^{-2 t} \ln \left(t^{2}+z_{3}^{2} \lambda(a)^{2}\right) d t-\ln \left(z_{3}\right)\right] .
$$

Hence

$$
2 \pi \frac{\operatorname{Im} w(z, z)}{\ln \left(z_{3}\right)}=-\lambda(a)+\frac{\lambda(a)\left(-\ln (\lambda(a))+\int_{0}^{\infty} e^{-2 t} \ln \left(t^{2}+z_{3}^{2} \lambda^{2}\right) d t\right)}{\ln \left(z_{3}\right)}
$$

which gives the formula:

$$
\lim _{z_{3} \rightarrow 0^{+}}-2 \pi \frac{\operatorname{Im} w(z, z)}{\ln \left(z_{3}\right)}=\lambda(a)
$$

For $m>3$, we use also the hyperspherical coordinates and get:

$$
w(z, z)=\frac{\sigma_{m-1}}{2(2 \pi)^{m-1}} \int_{0}^{\infty} e^{-2 z_{m} r} \frac{1}{2 r}\left(1+\frac{2 i \lambda(a)}{r-i \lambda(a)}\right) r^{m-1} d r .
$$

Hence

$$
\operatorname{Re} w(z, z)=\frac{\sigma_{m-1}}{2(2 \pi)^{m-1}} \frac{1}{\left(2 z_{m}\right)^{m-2}}+O\left(\frac{1}{\left(z_{m}\right)^{m-3}}\right)
$$

and

$$
\operatorname{Im} w(z, z)=\frac{\sigma_{m-1}}{(2 \pi)^{m-1}} \frac{\lambda(a)}{\left(2 z_{m}\right)^{m-3}}+O(1)
$$

This ends the proof of Proposition 3.1. 


\subsection{Proof of Proposition 3.2.}

We assume that the point $a$ is on $\partial D_{I}$. The case where $a$ is on $\partial D_{D}$ is similar and easier.

Let $\tilde{\Phi}^{s}$ be the corresponding solution as $\Phi^{s}$ replacing $\partial D_{I}$ by $\partial D$ (i.e. taking $\partial D_{D}=\emptyset$ ). We set $G_{\lambda}(x, z):=\tilde{\Phi}^{s}(x, z)+\Phi(x, z)$, the Green's function of the problem $(1.2),(1.3),(1.5)$. We set also $G_{\lambda(a)}(x, z)$ to be the Green's function of $(1.2),(1.3),(1.5)$ when the function $\lambda(x)$ is replaced by the constant function $\lambda(a)$. For both of the Green functions we assumed $\partial D_{D}=\emptyset$. Finally, we set $G_{\lambda(a)}^{0}$ to be the Green's function satisfying

$$
\left\{\begin{array}{l}
\Delta G_{\lambda}^{0}=-\delta \text { in } \Omega \backslash \bar{D} \\
\frac{\partial G_{\lambda}^{0}}{\partial \nu}(x, z)+i \lambda(a) G_{\lambda}^{0}(x, z)=0 \text { on } \partial D \\
G_{\lambda(a)}^{0}(\cdot, z)=0 \text { on } \partial \Omega
\end{array}\right.
$$

with an arbitrary fixed $C^{2}-$ regular domain $\Omega$ containing $\bar{D}$.

We have the following lemma

Lemma 3.4 For every $R>0$, there exists a positive constant $C:=C(R)$ such that

1. $\left|G_{\lambda}(x, z)\right| \leq \frac{C}{|x-z|^{m-2}}$,

2. $\left|\nabla G_{\lambda}(x, z)\right| \leq \frac{C}{|x-z|^{m-1}}$,

for $(x, z) \in\left(\mathbb{R}^{m} \backslash D\right) \cap B(0, R)$.

Proof of Lemma 3.4. These properties are known for general equations and boundary conditions. We refer to [19] and [20] where these results are justified for boundary value problems stated on bounded domains. Since the arguments are local, these estimates are also justified for exterior problems.

The function $\tilde{\Phi}^{s}-\Phi^{s}$ satisfies

$$
\left\{\begin{array}{l}
\left(\Delta+\kappa^{2}\right)\left(\tilde{\Phi}^{s}-\Phi^{s}\right)=0 \text { in } \mathbb{R}^{m} \backslash \bar{D} \\
\tilde{\Phi}^{s}-\Phi^{s}(x, z)=\tilde{\Phi}^{s}+\Phi \text { on } \partial D_{D} \\
\frac{\partial\left(\tilde{\Phi}^{s}-\Phi^{s}\right)}{\partial \nu}(x, z)+i \lambda(x)\left(\tilde{\Phi}^{s}-\Phi^{s}\right)(x, z)=0 \text { on } \partial D_{I} \\
\left(\tilde{\Phi}^{s}-\Phi^{s}\right)(\cdot, z) \text { satisfies Sommerfeld radiation conditions. }
\end{array}\right.
$$

For $z$ near $a$, Lemma 3.4 implies that $\left(\tilde{\Phi}^{s}+\Phi\right)(\cdot, z)$ is bounded in $H^{1 / 2}\left(\partial D_{D}\right)$. The well posedness of $(3.50)$, see [3], implies that $\left(\tilde{\Phi}^{s}-\Phi^{s}\right)(\cdot, z)$ is bounded in $H_{l o c}^{1}\left(\mathbb{R}^{m} \backslash \bar{D}\right)$. Introducing a cut off function around the point $a$ and knowing that $\tilde{\Phi}^{s}(\cdot, z)$ and $\Phi(\cdot, z)$ and their derivatives are bounded for $x$ near $\partial D_{D}$ and $z$ near $a$ (which is in $\partial D_{I}$ ), we deduce that $\left(\tilde{\Phi}^{s}-\Phi^{s}\right)$ is bounded for $x$ and $z$ near $a$. This implies that we can replace $\Phi^{s}$ by $\tilde{\Phi}^{s}$ in Proposition 3.2. In addition, by setting

$$
\tilde{\Phi}^{s}-\left(\Gamma_{\lambda(a)}-\Gamma\right)=G_{\lambda}-\Gamma_{\lambda(a)}-(\Phi-\Gamma),
$$

and knowing that $(\Phi-\Gamma)(x, z)$ is bounded in $\mathbb{R}^{m}$, then the proof of Proposition 3.2 is reduced to consider the term $G_{\lambda}-\Gamma_{\lambda(a)}$. We split the rest of the proof into the following three lemmas.

LEMMA 3.5 There exist $\delta(a)>0$ and $C(R)>0$ such that $\left|G_{\lambda}(x, z)-G_{\lambda(a)}(x, z)\right| \leq C(R) \mid z-$ $\left.a\right|^{3-m+\beta}+C(R)$, for $z \in B(a, \delta(a)) \cap C_{a, \theta}$ and $x \in \mathbb{R}^{m} \backslash D$.

LEMMA 3.6 There exists $C>0$ such that $\left|G_{\lambda(a)}(x, z)-G_{\lambda(a)}^{0}(x, z)\right| \leq C|z-a|^{4-m}+C$, for $z$ near $D$ and $x \in \Omega \backslash D$.

LEMMA 3.7 There exist $C>0$ and $\delta(a)>0$ such that

1. $\left|\operatorname{Im} G_{\lambda(a)}^{0}(x, z)-\operatorname{Im}_{\lambda(a)}(x, z)\right| \leq C|z-a|^{4-m}+C$, for $(x, z) \in B(a, \delta(a)) \cap C_{a, \theta}$.

2. $\left|\operatorname{Re} G_{\lambda(a)}^{0}(x, z)-\operatorname{Re}_{\lambda(a)}(x, z)\right| \leq C|\ln | z-a \|$ for $(x, z) \in B(a, \delta(a)) \cap C_{a, \theta}$, if $m=3$.

3. $\left|\operatorname{Re} G_{\lambda(a)}^{0}(x, z)-\operatorname{Re} \Gamma_{\lambda(a)}(x, z)\right| \leq C|z-a|^{3-m}$ for $(x, z) \in B(a, \delta(a)) \cap C_{a, \theta}$, if $m>3$. 
In the proofs of these last lemmas we do not, in general, specify the interdependency of the constants appearing in the estimates. However we distinguish the constant depending on the angle $\theta$ and the ones which do not depend.

Proof of Lemma 3.5. We set $R(x, z):=G_{\lambda}(x, z)-G_{\lambda(a)}(x, z)$. Then it satisfies:

$$
\left\{\begin{array}{l}
\left(\Delta+\kappa^{2}\right) R(x, z)=0 \text { in } \mathbb{R}^{m} \backslash \bar{D} \\
\frac{\partial R(x, z)}{\partial \nu}+i \lambda(a) R(x, z)=-i(\lambda-\lambda(a)) G_{\lambda}(x, z) \text { on } \quad \partial D \\
R(\cdot, z) \text { satisfies the Sommerfeld radiation condition. }
\end{array}\right.
$$

From (3.51), we have the representation:

$$
R(x, z)=-\int_{\partial D} i(\lambda(y)-\lambda(a)) G_{\lambda(a)}(y, x) G_{\lambda}(y, z) d s(y), \text { for }(x, z) \in \mathbb{R}^{m} \backslash \bar{D} .
$$

Hence letting $x$ tend to $\partial D$ we have:

$$
R(x, z)=-\int_{\partial D} i(\lambda(y)-\lambda(a)) G_{\lambda(a)}(y, x) G_{\lambda}(y, z) d s(y) \text { for } x \in \partial D \text { and } z \in \mathbb{R}^{m} \backslash \bar{D} .
$$

From the assumption on the regularity of the surface impedance $\lambda(x)$, we have

$$
|\lambda(y)-\lambda(a)| \leq C|y-a|^{\beta} .
$$

It is clear that $|y-a| \leq c(\theta)|y-z|$ for $y \in \partial D_{I}$ and $z \in C_{a, \theta} \cap B(a, \delta(a))$ with a positive constant $c(\theta)$ depending on the angle $\theta$. This is due to the fact that $\partial D_{I}$ and $C_{a, \theta} \cap B(a, \delta(a))$ are separated, i.e. $\partial D_{I} \cap C_{a, \theta} \cap B(a, \delta(a))=\{a\}$. From the inequality

$$
\frac{|\lambda(y)-\lambda(a)|}{|y-z|^{m-2}} \leq \frac{c(\theta)^{\beta} C}{|y-z|^{m-2-\beta}} .
$$

and the point 1) of Lemma 3.4, we have

$$
|R(x, z)| \leq \int_{\partial D} \frac{c(\theta)^{\beta} C}{|y-z|^{m-2-\beta}|y-x|^{m-2}} d y \leq \frac{C}{|x-z|^{m-3-\beta}}+C
$$

then

$$
\max _{x \in \partial D}|R(x, z)| \leq \frac{C}{|z-a|^{m-3-\beta}}+C \text { for } z \in C_{a, \theta} \cap B(a, \delta(a)) .
$$

Now, the solvability of the forward problem

$$
\left\{\begin{array}{l}
\left(\Delta+\kappa^{2}\right) R(x, z)=0 \text { in } \mathbb{R}^{m} \backslash \bar{D} \\
|R(\cdot, z)| \leq \frac{C}{|z-a|^{m-3-\beta}}+C \text { on } \partial D \\
R(\cdot, z) \text { satisfies the radiation conditions }
\end{array}\right.
$$

implies the desired estimate for $R(x, z)$ for $x \in \mathbb{R}^{m} \backslash D$ and $z \in C_{a, \theta} \cap B(0, R)$.

Proof of Lemma 3.6.

We recall that $G_{\lambda(a)}^{0}$ satisfies

$$
\left\{\begin{array}{l}
\Delta G_{\lambda}^{0}(x, z)=-\delta(x, z) \text { in } \Omega \backslash \bar{D} \\
\frac{\partial G_{\lambda}^{0}}{\partial \nu}(x, z)+i \lambda G_{\lambda}^{0}(x, z)=0 \text { on } \partial D \\
G_{\lambda}^{0}(x, z)=0 \text { on } \partial \Omega
\end{array}\right.
$$


Then, $G_{\lambda(a)}-G_{\lambda(a)}^{0}$ is solution of the problem

$$
\left\{\begin{array}{l}
\Delta\left(G_{\lambda(a)}-G_{\lambda(a)}^{0}\right)(x, z)=\kappa^{2} G_{\lambda(a)}(x, z) \text { in } \Omega \backslash \bar{D}, \\
\frac{\partial\left(G_{\lambda(a)}-G_{\lambda(a)}^{0}\right)}{\partial \nu}(x, z)+i \lambda(a)\left(G_{\lambda(a)}-G_{\lambda(a)}^{0}\right)(x, z)=0 \text { on } \partial D, \\
\left(G_{\lambda(a)}-G_{\lambda(a)}^{0}\right)(x, z)=G_{\lambda(a)}(x, z) \text { on } \partial \Omega .
\end{array}\right.
$$

Using integral representation for the solution of (3.55) and Lemma 3.4 applied for $G_{\lambda(a)}$ and $G_{\lambda(a)}^{0}$, we have the desired estimate for $\left(G_{\lambda(a)}-G_{\lambda(a)}^{0}\right)(x, z)$ for $x$ in $\Omega \backslash D$ and $z$ near $\partial D$.

Proof of Lemma 3.7. We can assume without loss of generality that $a=(0,0,0, \ldots, 0)$ by using a rigid transformation of coordinates. Let $\xi=F(x)$ be the following local change of variables

$$
\left\{\begin{array}{l}
\xi^{\prime}=x^{\prime} \\
\xi_{m}=x_{m}-f\left(x^{\prime}\right)
\end{array}\right.
$$

where $f$ is the function defined in the introduction. We have the following properties:

$$
\left\{\begin{array}{l}
c_{1}|x-z| \leq|F(x)-F(z)| \leq c_{2}|x-z| \\
|F(x)-x| \leq c_{3}|x|^{2} \\
|D F(x)-I| \leq c_{4}|x|
\end{array}\right.
$$

for $x, z$ near the point $a$, where $c_{i}, i=1, \ldots, 4$ are positive constants, which is due to the $C^{2}$ regularity of $\partial D$.

Let $x, z$ be near the point $a$. We set $\tilde{G}_{\lambda(a)}^{0}(\xi, \eta):=G_{\lambda(a)}^{0}(x, y)$, where $\xi=F(x)$ and $\eta:=F(z)$. Then $\tilde{G}_{\lambda(a)}^{0}(\cdot, \eta)$ satisfies:

$$
\left\{\begin{array}{l}
\nabla \cdot B(\xi) \nabla \tilde{G}_{\lambda(a)}^{0}=-\delta(\xi-\eta) \text { near } F(a) \\
\left|J^{-T} \nu\right| B \nabla \tilde{G}_{\lambda(a)}^{0} \cdot \nu+i \lambda(a) \tilde{G}_{\lambda(a)}^{0}=0 \text { on } \partial \mathbb{R}_{+}^{m} \text { near } F(a)
\end{array}\right.
$$

where $B=J J^{T}$ and $J=\frac{\partial \xi}{\partial x}\left(F^{-1}(\xi)\right)$. From the properties (3.57), we have

$$
|J(\xi)-J(0)| \leq c|\xi|,|B(\xi)-B(0)| \leq c|\xi|
$$

and $J(0)=B(0)=I$.

\section{First step}

Using similar notations as in the proof of Lemma 3.5 , we write $\tilde{R}(\xi, \eta)=\tilde{G}_{\lambda(a)}^{0}(\xi, \eta)-\Gamma_{\lambda(a)}(\xi, \eta)$, hence:

$$
\left\{\begin{array}{l}
\Delta \tilde{R}(\xi, \eta)=\nabla \cdot(I-B) \nabla \tilde{G}_{\lambda(a)}^{0} \text { near } F(a) \\
\nabla \tilde{R} \cdot \tilde{\nu}+i \lambda(a) \tilde{R}=(I-B) \nabla \tilde{G}_{\lambda(a)}^{0} \cdot \tilde{\nu}+i \lambda(a)\left(1-\left|J^{-T}\right|\right) \tilde{R} \text { on } \partial \mathbb{R}_{+}^{m} \text { near } F(a) .
\end{array}\right.
$$

Let $B_{r}^{+}:=B(F(a), r) \cap[F(D)]^{c}$ for $r$ small enough, then by (3.59) and using the local Green's function $\Gamma_{\lambda(a)}$, the solution $\tilde{R}$ has the following representation

$$
\begin{gathered}
-\tilde{R}(\xi, \eta)+\int_{\partial B_{r}^{+}} \frac{\partial \tilde{R}(z, \xi)}{\partial \nu} \Gamma_{\lambda(a)}(z, \eta) d s(z)-\int_{\partial B_{r}^{+}} \frac{\partial \Gamma_{\lambda(a)}(z, \eta)}{\partial \nu} \tilde{R}(z, \xi) d s(z) \\
=-\int_{B_{r}^{+}}(I-B) \nabla \tilde{G}_{\lambda(a)}^{0}(z, \xi) \cdot \nabla \Gamma_{\lambda(a)}(z, \eta) d z+\int_{\partial B_{r}^{+}}(I-B) \nabla \tilde{G}_{\lambda(a)}^{0}(z, \xi) \cdot \nu \Gamma_{\lambda(a)}(z, \eta) d s(z),
\end{gathered}
$$


for $\xi$ and $\eta$ in $B_{r}^{+}$. We write $\partial B_{r}^{+}=S_{r} \cup S_{r}^{c}$, where $S_{r}=\partial B_{r}^{+} \cap \partial(F(D))$. Using the impedance boundary condition on $S_{r}$, the last equation becomes

$$
\begin{gathered}
-\tilde{R}(\xi, \eta)-\int_{S_{r}} i \lambda(a) \tilde{R}(z, \xi) \cdot \Gamma_{\lambda(a)}(z, \eta) d s(z)+\int_{S_{r}} i \lambda(a) \Gamma_{\lambda(a)}(z, \eta) \tilde{R}(z, \xi) d s(z) \\
=-\int_{B_{r}^{+}}(I-B) \nabla \tilde{G}_{\lambda(a)}^{0}(z, \eta) \cdot \nabla \Gamma_{\lambda(a)}(z, y) d z+\int_{\partial B_{r}^{+}}(I-B) \nabla \tilde{G}_{\lambda(a)}^{0}(z, \xi) \cdot \nu \Gamma_{\lambda(a)}(z, \eta) d s(z) \\
\quad+\int_{S_{r}^{c}} \frac{\partial}{\partial \nu} \tilde{R}(z, \xi) \Gamma_{\lambda(a)}(z, \eta) d s(z)+\int_{S_{r}^{c}} \frac{\partial}{\partial \nu} \Gamma_{\lambda(a)}(z, \eta) \tilde{R}(z, \xi) d s(z) \\
-\int_{S_{r}}(I-B) \nabla \tilde{G}_{\lambda(a)}^{0}(z, \xi) \cdot \nu \Gamma_{\lambda(a)}(z, \eta) d s(z)-i \lambda(a) \int_{S_{r}}\left(1-\left|J^{-T} \nu\right|\right) \tilde{R}(z, \eta) \Gamma_{\lambda(a)}(z, \xi) d s(z)
\end{gathered}
$$

After simplification we have

$$
-\tilde{R}(\xi, \eta)=-\int_{B_{r}^{+}}(I-B) \nabla \tilde{G}_{\lambda(a)}^{0}(z, \xi) \cdot \nabla \Gamma_{\lambda(a)}(z, \eta) d z+\int_{S_{r}^{c}}(I-B) \frac{\partial \tilde{G}_{\lambda(a)}^{0}(z, \xi)}{\partial \nu} \Gamma_{\lambda(a)}(z, \eta) d s(z)
$$

$+\int_{S_{r}^{c}} \frac{\partial \tilde{R}}{\partial \nu}(z, \xi) \Gamma_{\lambda(a)}(z, \eta) d s(z)+\int_{S_{r}^{c}} \frac{\partial}{\partial \nu} \Gamma_{\lambda(a)}(z, \eta) \tilde{R}(z, \xi) d s(z)-i \lambda(a) \int_{S_{r}}\left(1-\left|J^{-T} \nu\right|\right) \tilde{R}(z, \eta) \Gamma_{\lambda(a)}(z, \xi) d s(z)$.

Taking the imaginary part in the last equality, we have

$$
\begin{gathered}
-\int_{B_{r}^{+}}(I-B) \nabla\left(\operatorname{Re} \tilde{G}_{\lambda(a)}^{0}\right)(z, \xi) \cdot \nabla\left(\operatorname{Im} \Gamma_{\lambda(a)}\right)(z, \eta) d z \\
+\int_{S_{r}^{c}}(I-B) \frac{\partial \operatorname{Im} \tilde{G}_{\lambda(a)}^{0}}{\partial \nu}(z, \xi) \operatorname{Re} \Gamma_{\lambda(a)}(z, \eta) d s(z)+\int_{S_{r}^{c}}(I-B) \frac{\partial \operatorname{Re} \tilde{G}_{\lambda(a)}^{0}}{\partial \nu}(z, \xi) \operatorname{Im} \Gamma_{\lambda(a)}(z, \eta) d s(z) \\
+\int_{S_{r}^{c}} \frac{\partial \operatorname{Im} \tilde{R}}{\partial \nu}(z, \xi) \operatorname{Re} \Gamma_{\lambda(a)}(z, \eta) d s(z)+\int_{S_{r}^{c}} \frac{\partial \operatorname{Re} \tilde{R}}{\partial \nu}(z, \xi) \operatorname{Im} \Gamma_{\lambda(a)}(z, \eta) d s(z) \\
+\int_{S_{r}^{c}} \frac{\partial}{\partial \nu} \operatorname{Im} \Gamma_{\lambda(a)}(z, \eta) \operatorname{Re} \tilde{R}(z, \xi) d s(z)+\int_{S_{r}^{c}} \frac{\partial}{\partial \nu} \operatorname{Re} \Gamma_{\lambda(a)}(z, \eta) \operatorname{Im} \tilde{R}(z, \xi) d s(z) \\
-\lambda(a) \int_{S_{r}}\left(1-\left|J^{-T} \nu\right|\right) \operatorname{Re}\left[\tilde{R}(z, \eta) \Gamma_{\lambda(a)}(z, \xi)\right] d s(z) .
\end{gathered}
$$

We have for $\Gamma_{\lambda(a)}$ similar estimates as in Lemma 3.4. In particular, we have

$$
\left|\nabla\left(\operatorname{Re} \Gamma_{\lambda(a)}\right)(x, z)\right| \leq c|x-z|^{1-m} .
$$

It is of importance to remark that the imaginary parts have less singularities. Indeed, we will prove the following lemma:

Lemma 3.8 For every $R>0$, there exists $c:=c(R)$ such that

$\left|\nabla\left(\operatorname{Im} \Gamma_{\lambda(a)}\right)(x, z)\right| \leq c|x-z|^{2-m+s} d^{-s}\left(x, \partial B_{+}\right)$and $\left|\nabla\left(\operatorname{Im} \tilde{G}_{\lambda(a)}^{0}\right)(x, z)\right| \leq c|x-z|^{2-m+s} d^{-s}\left(x, \partial B_{+}\right)$ for $x, z$ in $B_{+}(0, R):=B(0, R) \cap \mathbb{R}_{+}^{m}$. 
Proof of Lemma 3.8.

From (3.26), we deduce that $\operatorname{Im} \Gamma_{\lambda(a)}(\cdot, z)$ satisfies

$$
\left\{\begin{array}{l}
\Delta\left(\operatorname{Im} \Gamma_{\lambda(a)}\right)(\cdot, z)=0, \text { in } \mathbb{R}_{+}^{m} \\
\frac{\partial}{\partial \nu} \operatorname{Im} \Gamma_{\lambda(a)}(\cdot, z)=-\lambda(a) \operatorname{Re} \Gamma_{\lambda(a)}(\cdot, z) \text { on } \partial \mathbb{R}_{+}^{m}
\end{array}\right.
$$

Let $\Omega$ be a regular domain in $\mathbb{R}^{m}$ symmetric with respect to the plane $\left\{x_{m}=0\right\}$. We state the problem (3.62) on $\Omega_{+}:=\Omega \cap \mathbb{R}_{+}^{m}$. Let $G_{+}$be the Neumann Green function of the Laplace on $\Omega_{+}$. From (3.62) we can write

$$
\operatorname{Im} \Gamma_{\lambda(a)}(x, z)=\int_{\partial \Omega_{+}} G_{+}(x, y) \frac{\partial \operatorname{Im} \Gamma_{\lambda(a)}}{\partial \nu}(z, y) d s(y) .
$$

The boundary condition in $(3.62)$ on $\partial \Omega_{+} \cap \partial \mathbb{R}_{+}^{m}$ gives

$\operatorname{Im} \Gamma_{\lambda(a)}(x, z)=-\int_{\partial \Omega_{+} \cap \partial \mathbb{R}_{+}^{m}} \lambda(a) G_{+}(x, y) \operatorname{Re} \Gamma_{\lambda(a)}(z, y) d s(y)-\int_{\partial \Omega_{+} \backslash \partial \mathbb{R}_{+}^{m}} G_{+}(x, y) \frac{\partial \operatorname{Im} \Gamma_{\lambda(a)}}{\partial \nu}(z, y) d s(y)$.

Taking the derivatives, we have

$$
\begin{gathered}
\nabla_{x}\left(\operatorname{Im} \Gamma_{\lambda(a)}\right)(x, z)=-\int_{\partial \Omega_{+} \cap \partial \mathbb{R}_{+}^{m}} \lambda(a) \nabla_{x} G_{+}(x, y) \operatorname{Re} \Gamma_{\lambda(a)}(z, y) d s(y)- \\
-\int_{\partial \Omega_{+} \backslash \partial \mathbb{R}_{+}^{m}} \nabla_{x} G_{+}(x, y) \frac{\partial \operatorname{Im} \Gamma_{\lambda(a)}}{\partial \nu}(z, y) d s(y) .
\end{gathered}
$$

Taking $\Omega$ large enough to contain $B_{+}(0, R)$ and using the estimates of $G_{+}$and $\Gamma_{\lambda(a)}$ given in Lemma 3.4, we deduce that the second term in the right hand side is bounded for $x, z$ in $B_{+}(0, R)$ because $\partial \Omega_{+} \backslash \partial \mathbb{R}_{+}^{m}$ is away from $B_{+}(0, R)$. The first term can be estimated by

$$
\begin{aligned}
& \left|\int_{\partial \Omega_{+} \cap \partial \mathbb{R}_{+}^{m}} \lambda(a) \nabla_{x} G_{+}(x, y) \operatorname{Re} \Gamma_{\lambda(a)}(z, y) d s(y)\right| \leq c\left|\int_{\partial \Omega_{+} \cap \partial \mathbb{R}_{+}^{m}}\right| x-\left.y\right|^{1-m}|y-z|^{2-m} d s(y) \mid \\
& \quad \leq c\left|\int_{\partial \Omega_{+} \cap \partial \mathbb{R}_{+}^{m}}\right| x-\left.y\right|^{1-m+s}|y-z|^{2-m} d s(y) d^{-s}\left(x, \partial \Omega_{+}\right) \leq c_{s}|x-z|^{2-m+s} d^{-s}\left(x, \partial \Omega_{+}\right)
\end{aligned}
$$

where $c_{s}$ is a positive constant behaving as $\frac{1}{1-s}$, see [14] (or [21] for Lipschitz surfaces). Similar arguments can be applied for $\tilde{G}_{\lambda(a)}^{0}$.

Using this lemma and the estimates of $\tilde{G}_{\lambda(a)}^{0}$, in $(3.61)$, we have

$$
\begin{gathered}
|\operatorname{Im} \tilde{R}(\xi, \eta)| \leq c\left[\int_{B_{r}^{+}}|z| d^{-s}\left(z, \partial B_{r}^{+}\right)|z-\xi|^{2-m+s}|z-\eta|^{1-m} d z+\int_{B_{r}^{+}}|z||z-\xi|^{1-m} d^{-s}\left(z, \partial B_{r}^{+}\right)|z-\eta|^{2-m+s} d z+\right. \\
\int_{S_{r}^{c}} d^{-s}\left(z, \partial B_{r}^{+}\right)|z-\xi|^{2-m+s}|z-\eta|^{1-m} d s(z)+\int_{S_{r}^{c}}|z-\xi|^{1-m} d^{-s}\left(z, \partial B_{r}^{+}\right)|z-\eta|^{2-m+s} d s(z)+ \\
\left.\int_{S_{r}}|z||z-\xi|^{2-m}|z-\eta|^{2-m} d s(z)\right]
\end{gathered}
$$

where $c$ is a positive constant independent on $\xi$ and $\eta$.

Taking $\eta \in C_{F(a), \theta}$ and $\xi$ on $S_{r}$ away from $S_{r}^{c}$, from (3.65) we will show the desired estimate of $\operatorname{Im} \tilde{R}(\xi, \eta)$. 
The third and the forth integrals in (3.65) are bounded because $\xi$ is away from $S_{r}^{c}$. Let us consider the first integral. For $\eta \in C_{F(a), \theta}$ and $\xi$ on $S_{r}$, we have the inequality

$$
|\xi| \leq c(\theta)|\xi-\eta|
$$

with some positive constant $c(\theta)$. We decompose $B_{r}^{+}$as $B_{r}^{+}=B_{r_{1}}^{+} \cup B_{r_{2}}^{+}$, where $B_{r_{1}}^{+}:=B_{r}^{+} \cap C_{F(a), \tilde{\theta}}$ where $\theta<\tilde{\theta}<\frac{\pi}{2}$ and $B_{r_{2}}^{+}:=B_{r}^{+} \backslash B_{r_{1}}^{+}$.

Let us consider $\int_{B_{r_{1}}^{+}}|z| d^{-s}\left(z, \partial B_{r}^{+}\right)|z-\xi|^{2-m+s}|z-\eta|^{1-m} d z$. For $z \in B_{r}^{+}$, we have $|z| \leq$ $c(\tilde{\theta}) d\left(z, \partial B_{r}^{+}\right)$for some positive constant $c(\tilde{\theta})$. Hence we need to consider the integral:

$$
\int_{B_{r_{1}}^{+}}|z|^{1-s}|z-\xi|^{2-m+s}|z-\eta|^{1-m} d z
$$

At this point, we make use of an argument from ([1], pages 209, 210). We decompose the last integral as the sum of

$$
I_{1}(\xi, \eta):=\int_{\{|z|<4 c(\theta) h\} \cap B_{r_{1}}^{+}}|z|^{1-s}|z-\xi|^{2-m+s}|z-\eta|^{1-m} d z
$$

and

$$
I_{2}(\xi, \eta):=\int_{\{|z|>4 c(\theta) h\} \cap B_{r_{1}}^{+}}|z|^{1-s}|z-\xi|^{2-m+s}|z-\eta|^{1-m} d z
$$

where $h:=|\xi-\eta|$. After the change of variables $t:=\frac{z}{h}$, we obtain

$$
I_{1}(\xi, \eta) \leq h^{4-m} 4 c(\theta) \int_{|t|<4 c(\theta)}|t|^{1-s}\left|\frac{\xi}{h}-t\right|^{2-m+s}\left|\frac{\eta}{h}-t\right|^{1-m} d t .
$$

Since $\left|\frac{\xi}{h}-\frac{\eta}{h}\right|=1$, then from ([14], Chapter 2, section 11), the integral in the left hand side is bounded. Hence

$$
I_{1}(\xi, \eta) \leq c|\xi-\eta|^{4-m}
$$

Let us now consider the term $I_{2}(\xi, \eta)$. From the inequality (3.66), we have

$$
|z| \leq|z-\xi|+|\xi| \leq|z-\xi|+c(\theta)|h| \leq|z-\xi|+\frac{1}{4}|z|,
$$

hence

$$
|z| \leq \frac{4}{3}|z-\xi|
$$

Similarly we get $|z| \leq \frac{4 c(\theta)}{3 c(\theta)-1}|z-\eta|$. In (3.66), we can always take $c(\theta)>\frac{1}{3}$. Hence

$$
I_{2}(\xi, \eta) \leq c \int_{r>|z|>4 c(\theta) h}|z|^{4-2 m} d z \leq C|\xi-\eta|^{4-m}+C .
$$

Summing up (3.67) and (3.69), implies

$$
\int_{B_{r_{1}}^{+}}|z| d^{-s}\left(z, \partial B_{r}^{+}\right)|z-\xi|^{2-m+s}|z-\eta|^{1-m} d z \leq C|\xi-\eta|^{4-m}+C .
$$


Now, we deal with $\int_{B_{r_{2}}^{+}}|z| d^{-s}\left(z, \partial B_{r}^{+}\right)|z-\xi|^{2-m+s}|z-\eta|^{1-m} d z$. In this case $d\left(z, \partial B_{r_{2}}^{+}\right)=\left|z_{m}\right|$. Since $\eta \in C_{F(a), \theta}$ then $|z-\eta|^{1-m}>c>0$, since $C_{F(a), \theta}$ and $B_{r_{2}}^{+}$are separated sets.

Hence using these informations with the fact that $|z| \leq \frac{4}{3}|z-\xi|$, we have

$$
\int_{B_{r_{2}}^{+}}|z| d^{-s}\left(z, \partial B_{r}^{+}\right)|z-\xi|^{2-m+s}|z-\xi|^{1-m} d z \leq \int_{B_{r_{2}}^{+}}|z|^{3-m+s}\left|z_{m}\right|^{-s} d z .
$$

By using the Holder inequality and choosing $s$ small enough we have $\int_{B_{r_{2}}^{+}}|z|^{3-m+s}\left|z_{m}-\xi\right|^{s} d z<\infty$. This means that the first integral of (3.65) is estimated by $C|\xi-\eta|^{4-m}+C$.

Arguing as before for the first terms of (3.65), we deduce that the second term has similar estimate. From the inequality (3.68), the last integral is bounded by $c \int_{S_{r}}|z-\xi|^{3-m}|z-\eta|^{2-m} d s(z)$ which is itself bounded by $C|\xi-\eta|^{4-m}+C$. Finally we deduce that the integral in (3.65) is also bounded by $C|\xi-\eta|^{4-m}+C$.

\section{Second step}

We go back to estimate $R(x, z):=G_{\lambda(a)}^{0}(x, z)-\Gamma_{\lambda(a)}(x, z)$. We have

$$
R(x, z)=G_{\lambda(a)}^{0}(x, z)-\Gamma_{\lambda(a)}(F(x), F(z))+\Gamma_{\lambda(a)}(F(x), F(z))-\Gamma_{\lambda(a)}(x, z)
$$

which we write as

$(3.71)$

$$
R(x, z)=\tilde{R}(F(x), F(z))+\left(\Gamma_{\lambda(a)}(F(x), F(z))-\Gamma_{\lambda(a)}(F(x), z)\right)+\left(\Gamma_{\lambda(a)}(F(x), z)-\Gamma_{\lambda(a)}(x, z)\right) .
$$

Let us show that $F\left(C_{a, \theta} \cap B(0, \delta(a))\right) \subset C_{F(a), \theta^{\prime}}$ for some $\theta^{\prime} \in(0, \pi / 2)$ and $\theta \leq \theta^{\prime}$ with $\delta(a)$ small enough. Recall that $a=(0,0,0)$. We set $M:=\max _{\left(z_{1}, z_{2}\right) \in \overline{B_{2}}(0, \delta(a))}\left|f\left(z_{1}, z_{2}\right)\right|=\left|f\left(z_{1}^{0}, z_{2}^{0}\right)\right|$. We draw the cone with vertex $a$, axis $(-\nu(a))$ and having the point $\left(z_{1}^{0}, z_{2}^{0}, M\right)$ on its boundary. We denote by $\phi$ the angle between this cone and the plan $\left\{z_{3}=0\right\}$. Since $|\nabla f(0,0)|=0$ and $f \in C^{1}(0, \delta(a))$, then $\phi=\phi(\delta(a))$ tends to zero if $\delta(a)$ tends to zero. Hence taking $\delta(a)$ small enough, if necessary, we can assume the $0 \leq \phi<\pi / 2-\theta$. Finally we define the cone with center $a$, axis $(-\nu(a))$ and angle $\theta^{\prime}:=\phi+\theta$. Then $F\left(C_{a, \theta}\right) \subset C_{F(a), \theta^{\prime}}$.

Let $x$ be near $a$ such that $F(x) \in S_{r}$ and away from $S_{r}^{c}$ and $z \in C_{a, \theta} \cap B(a, \delta(a))$. Then $(F(x), F(z)) \in S_{r} \times C_{F(a), \theta^{\prime}}$ and hence

$$
\operatorname{Im} \tilde{R}(F(x), F(z)) \leq C|F(x)-F(z)|^{4-m}+C \leq C|x-z|^{4-m}+C
$$

as shown in the first step.

In the following we need to estimate the second and the third terms of the right hand side of (3.71). We write

$$
\left|\Gamma_{\lambda(a)}(F(x), F(z))-\Gamma_{\lambda(a)}(F(x), z)\right| \leq\left|\nabla_{z} \operatorname{Im} \Gamma_{\lambda(a)}(F(x), \cdot)\right|_{L^{\infty}\left(V_{z, \epsilon}\right)}|F(z)-z|
$$

and

$$
\left|\Gamma_{\lambda(a)}(F(x), z)-\Gamma_{\lambda(a)}(x, z)\right| \leq\left|\nabla_{x} \operatorname{Im} \Gamma_{\lambda(a)}(\cdot, z)\right|_{L^{\infty}\left(V_{x, \epsilon}\right)}|F(x)-x|
$$

where $V_{z, \epsilon}$ is an open set containing $z$ and $F(z)$ such that $F(x) \in V_{z, \epsilon}^{c}$ and $V_{x, \epsilon}$ is an open set containing $x$ and $F(x)$ such that $z \in V_{x, \epsilon}^{c}$

From the representation (3.64) in the proof of Lemma 3.8, we have

$$
\left|\nabla_{z}\left(\operatorname{Im} \Gamma_{\lambda(a)}(F(x), \cdot)\right)\right|_{L^{\infty}\left(V_{z, \epsilon}\right)}<c\left[d\left(F(x), \partial V_{z, \epsilon}\right)\right]^{2-m}
$$


and similarly, we have

$$
\left|\nabla_{x}\left(\operatorname{Im} \Gamma_{\lambda(a)}(\cdot, z)\right)\right|_{L^{\infty}\left(V_{x, \epsilon}\right)}<c\left[d\left(z, \partial V_{x, \epsilon}\right)\right]^{2-m}
$$

where $c$ is independent of $V_{z, \epsilon}$ and $V_{x, \epsilon}$.

Hence (3.72) and (3.73) become

$$
\left|\Gamma_{\lambda(a)}(F(x), F(z))-\Gamma_{\lambda(a)}(F(x), z)\right| \leq c\left[d\left(F(x), \partial V_{z, \epsilon}\right)\right]^{2-m}|F(z)-z|
$$

and

$$
\left|\Gamma_{\lambda(a)}(F(x), z)-\Gamma_{\lambda(a)}(x, z)\right| \leq c\left[d\left(z, \partial V_{x, \epsilon}\right)\right]^{2-m}|F(x)-x|
$$

respectively. Since $\epsilon>0$ is arbitrary, we deduce, by shrinking $V_{z, \epsilon}$ to tend to a line joining the points $z$ and $F(z)$, and similarly $V_{x, \epsilon}$ to tend to a line joining the points $x$ and $F(x)$, that there exists $c>0$ such that

$$
\left\{\begin{array}{l}
\left|\Gamma_{\lambda(a)}(F(x), F(z))-\Gamma_{\lambda(a)}(F(x), z)\right| \leq c|F(x)-F(z)|^{2-m}|F(z)-z| \\
\quad \text { or } \\
\left|\Gamma_{\lambda(a)}(F(x), F(z))-\Gamma_{\lambda(a)}(F(x), z)\right| \leq c|F(x)-z|^{2-m}|F(z)-z| .
\end{array}\right.
$$

and

$$
\left\{\begin{array}{l}
\left|\Gamma_{\lambda(a)}(F(x), z)-\Gamma_{\lambda(a)}(x, z)\right| \leq c|x-z|^{2-m}|F(x)-x| \\
\text { or } \\
\left|\Gamma_{\lambda(a)}(F(x), z)-\Gamma_{\lambda(a)}(x, z)\right| \leq c|F(x)-z|^{2-m}|F(x)-x|
\end{array}\right.
$$

Reminding

$$
|x| \leq c(\theta)|x-z|
$$

for $x$ near $a$ and $z \in C_{a, \theta} \cap B(a, \delta(a))$ and using (3.57), we have

$$
|x-z| \leq|x-F(x)|+|F(x)-z| \leq c|x|^{2}+|F(x)-z| \leq c(\theta)|x-z|^{2}+|F(x)-z| .
$$

Hence

$$
|x-z|[1-c(\theta)|x-z|] \leq|F(x)-z| .
$$

Taking $\delta(a)$ small enough so that we have $\left(1-c(\theta)|x-z|^{2}\right) \geq 1 / 2$, then

$$
|x-z| \leq 2|F(x)-z| \text {. }
$$

From (3.57), we have

$$
|F(x)-x| \leq c|x|^{2} \leq c(\theta)|x-z|^{2},
$$

and also

$$
|F(z)-z| \leq c|z|^{2} \leq c(\theta)|x-z|^{2},
$$

Since $|x-z| \leq c|F(x)-F(z)|$ then using (3.76) and (3.77) in (3.74) and (3.75) we deduce that the second and the third terms of (3.71) are estimated by $C|x-z|^{4-m}$.

This means that

$$
|\operatorname{Im} R(x, z)| \leq C|x-z|^{4-m}+C
$$

for $x \in B(a, \delta(a))$ such that $F(x) \in S_{r}$ and $z \in C_{a, \theta} \cap B(a, \delta(a))$. 
For $z \in C_{a, \theta} \cap B\left(a, \frac{\delta(a)}{2}\right)$ and $x \in[\partial B(a, \delta(a))] \cap \mathbb{R}^{m} \backslash \bar{D}$, we have

$$
|\operatorname{Im} R(x, z)| \leq C
$$

with some positive constant $c$, because $C_{a, \theta} \cap B\left(a, \frac{\delta(a)}{2}\right)$ and $\left.\partial B(a, \delta(a))\right] \cap \mathbb{R}^{3} \backslash \bar{D}$ are separated sets. Since in $B(a, \delta(a)) \cap\left(\mathbb{R}^{3} \backslash \bar{D}\right)$, we have $\Delta_{x} \operatorname{Im} R(x, z)=0$, then using (3.78) and (3.79) we have, by the maximum principle,

$$
|\operatorname{Im} R(x, z)| \leq c(\theta)|z-a|^{4-m}+C
$$

for $x \in\left[\mathbb{R}^{3} \backslash D\right] \cap B(a, \delta(a))$ and $z \in C_{a, \theta} \cap B\left(a, \frac{\delta(a)}{2}\right)$.

Considering the real part of $R(x, z)$, by similar arguments as for $\operatorname{Im} R(x, z)$, we prove that

$$
|\operatorname{Re} R(x, z)| \leq\left\{\begin{array}{l}
c(\theta)|\ln | z-a||, \text { if } m=3 \\
c(\theta) \frac{1}{|z-a|^{m-3}} \text { if } m>3 .
\end{array}\right.
$$

This ends the proof of lemma 3.7 .

ACKNOWLEDGMENT. The first author is partially supported by Japan Society for promotion of Science. The second author is supported by Impedance Imaging Research Center of Kyung Hee University, Korea, via MOST/KOSEF (R11-2002-103) and SFB-project F1308 at RICAM, Austria.

\section{References}

[1] G. Alessandrini, M. Di Cristo, Stable Determination of an Inclusion by Boundary Measurements. SIAM Journal on Mathematical Analysis Volume 37, Number 1 pp. 200-217, (2005).

[2] I. Akduman, R. Kress, Direct and inverse scattering problems for inhomogeneous impedance cylinders of arbitrary shape. Radio Science 38, 1055-1064 (2003).

[3] F. Cakoni and D. Colton, The determination of the surface impedance of a partially coated obstacle from far field data. SIAM. J. Appl. Math.; 64, 709-723, (2004).

[4] F. Cakoni and D. Colton, Open problems in the qualitative approach to inverse electromagnetic scattering theory. European Journal of Applied Mathematics; 16, N 1: 1-15, (2005).

[5] F. Cakoni, D. Colton, Qualitative Methods in Inverse scattering Theory, Interaction of Mechanics and Mathematics, Springer, 2006.

[6] D. Colton and R. Kress, Inverse Acoustic and Electromagnetic Scattering Theory. 2nd edition (Berlin-Springer) (1998).

[7] J. Cheng, J. Liu and G. Nakamura, Recovery of Boundaries and Types of Multiple Obstacles from Far Field Pattern. Preprint.

[8] H. W. Engl, M. Hanke and A. Neubauer, Regularization of Inverse Problems. Mathematics and its Applications, 375, Kluwer Academic Publishers, Dorderecht/Boston/London, (1996).

[9] V. Isakov, Inverse Problems for Partial Differential Equations. Springer Series in Applied Math. Science. Berlin: Springer, 127, (1998). 
[10] A. Kirsch, An introduction to the Mathematical Theory of Inverse Problems, Springer Series in Applied Mathematical Sciences, 120, (1996).

[11] R. Kress, W. Rundell, Inverse scattering for shape and impedance. Inverse Problems; 17, 1075-1085, (2001).

[12] C. Labreuche, Stability of the recovery of surface impedances in inverse scattering. J. Math. Anal. Appl.; 231, 1, 161-176, (1999).

[13] J. Liu, G. Nakamura, M. Sini, Reconstruction of the shape and surface impedance from acoustic scattering data for arbitrary cylinder. Siam. J. Applied. Math. At press.

[14] C. Miranda, Partial differential equations of elliptic type. Springer-Verlag, New York-Berlin, (1970).

[15] W. McLean, Strongly Elliptic Systems and Boundary Integral Equations. Cambridge University press, (2000).

[16] R. Potthast, Point sources and multipoles in inverse scattering theory, V 427 of ChapmanHall/CRC, Research Notes in Mathematics. Chapman-Hall/CRC, Boca Raton, Fl, (2001).

[17] R. Potthast, Sampling and Probe Methods - An Algorithmical View. Computing, 75, no. 2-3, 215-235, (2005).

[18] E. Sincich, Stable determination of the surface impedance of an obstacle by far field measurements, Siam J. Math. Anal.; 38, 2, 434-451, (2006).

[19] V. A. Solonnikov, On Green's matrices for elliptic boundary value problems. I Proc. Steklov. Inst. Math, 110 (1970).

[20] V. A. Solonnikov, The Green's matrices for elliptic boundary value problems. II. Boundary value problems of mathematical physics, 7. Trudy. Math. Inst. Steklov. 116, 181-216 (1971).

[21] N. Valdivia, Uniqueness in inverse obstacle scattering with conductive boundary condictions. Appl. Anal. 83, no. 8, 825-851, (2004). 